\title{
SESSION 8
}

\section{STRUCTURE AND ROLE OF EMERGING FLUX REGIONS}




\title{
STRUCTURE OF EMERGING FLUX REGIONS
}

\section{DEAN-YI CHOU}

Department of Physics, Tsing Hua University Hsinchu, 30043, Taiwan

\begin{abstract}
We review the present status of our understanding of the structure and dynamics of emerging flux regions which form the first stage of active regions. We review the observations of the emerging flux regions, and discuss their structure, dynamics, and physical conditions derived from these observations. We also discuss implications about magnetic fields below the surface.
\end{abstract}

\section{EMERGENCE OF MAGNETIC FIELDS}

Observations give evidence that magnetic fields on the Sun emerge from below. In the traditional picture of the solar dynamo, magnetic fields are generated in the convection zone through the interaction of solar rotation and convective motions (Parker 1955, 1979; Babcock 1961; Leighton 1969;, Yoshimura 1975; Gilman and Miller 1981; Durney and Robinson 1982). This convection zone dynamo mechanism gives a numbers of problems and inconsistences. A numbers of authors have suggested that the site of solar dynamo action is not distributed through the convection zone, but lies instead in the convective overshoot region, a thin, convectively stable layer just below the convection zone (Rosner 1980; Spiegel and Weiss 1980; Golub et al. 1981; Galloway and Weiss 1981; van Ballegooijen 1982; Schmitt and Rosner 1983; Deluca and Gilman 1987; Gilman et al. 1989). Magnetic fields generated in the convective overshoot region are brought to the surface by magnetic buoyancy. The emergence of anchored magnetic flux through the convection zone has been studied by Babcock (1961), Leighton (1969), Schussler (1977, 1979), Parker $(1979,1987 \mathrm{a}, \mathrm{b}, \mathrm{c})$, Moreno-Insertis $(1983,1986)$, Choudhuri and Gilman (1987), Chou and Fisher (1989), and Fisher et al. (1989)

Emerging magnetic fields always appear in bipolar form at the surface, known as emerging flux regions (EFR's). The first signature of new emerging magnetic fields is a small, bright $\mathrm{H} \alpha$ region(Bumba and Howard 1965; Weart and Zirin 1969; Born 1974; Glackin 1975; Kawaguchi and Kitai 1976, Zirin 1988). EFR's also show as a compact bright plage in Ca II K-line and an intense X-ray source (Harvey et al. 1975; Golub et al. 1976, 1977; Moore et al. 1977; Sheeley and Golub 1979). Sometimes surges and small flares are observed in EFR's (Marsh 1978; Kurokawa 1988, 1991; Zirin 1988). Since the 
magnetic fields are almost horizontal at this stage, they appear very weak in magnetograms. If the amount of emerging flux is greater than about $10^{20}$ Maxwells, the arch filament systems (AFS's) appear (Harvey and Martin 1973; Chou and Wang 1987). AFS's, which are believed to trace out loops of the emerging magnetic fields, connect the opposite polarity fields (Weart and Zirin 1969; Weart 1972). Most new regions stop growing in less than a day, but sometimes magnetic flux continue to emerge and a simple bipolar region may grow into a full-fledged active region. Although ephemeral regions and EFR's may have different origins, there is no clear cut between them in terms of observed quantities, such as size, flux, and magnetic field strength (Harvey and Martin 1973; Chou and Wang 1987). The pores form in two opposite polarities if the emerging flux is greater than about $10^{20}$ Maxwells. As more flux keeps emerging, more pores form and coalesce to form sunspots (Vrabec 1974; Wang and Zirin 1992).

As magnetic fields emerge, the opposite poles move apart with a separation velocity of about $0.2-1.2 \mathrm{~km} \mathrm{~s}^{-1}$ (Chou and Wang 1987). The motion of preceding poles account for most of the separation; the following poles are almost motionless. The separation velocity drops to about $0.1-$ $0.2 \mathrm{~km} \mathrm{~s}^{-1}$ in the next few days (Mosher 1977).

EFR's carry a lot of informations about the magnetic fields in the convection zone. First, theoretical studies show that the rise time of a magnetic flux tube through the convection zone depends on its cross section and field strength, and the separation of its footpoints if it is anchored in the convective overshoot region (Parker 1979; Moreno-Insertis 1983, 1986; Chou and Fisher 1989). Because of the long journey, the dispersion of calculated rise time of magnetic flux tubes is much longer than observed time scales of active regions formation. It suggests that active regions form from the emergence of a single flux tube (Fisher et al. 1989). The observed fragmentation into small discrete flux tubes in active regions might be caused by the convection or other instabilities near the surface. It has been doubted whether the emerging flux is appreciably affected by the convection, such as the supergranulation ( $Z$ waan 1985). In any case, there are conflicting reports regarding where magnetic fields emerge with respect to the supergranulation or the chromospheric network (Bumba and Howard 1965; Frazier 1972; Harvey and Martin 1973; Schoolman 1973; Born 1974; Zirin 1974); Zwaan (1985) has argued that it is not trivial to compare the position of the emerging flux with the position of the previous supergranules or the chromospheric network. We argue that even if the emerging flux were locally affected by the convection, the fact that the separation of two poles eventually increases to a value greater than the size of supergranules suggests that the convection of supergranules does not play a major role in the large-scale emergence of magnetic fields. Moreover, initially the emerging bipoles can have any orientation, but they quickly rotate until they line up close to the East-West direction (Weart 1970). This also indicates that the large-scale evolution of EFR's is not affected by the local environment, such as convection; instead they are controlled by their own dynamics.

The lithium abundance of arch filaments (AF's) could provide the information on the seat of dynamo. As a flux tube emerges through the convection zone, the material inside the flux tube does not mix up with the surrounding material because the magnetic diffusivity is low. The abundance in AF's is expected to be the same as that in the region where the flux tube 
forms. Lithium is believed to be burned up at a temperature of about $2.4 \times 10^{6}$ degrees, which is close to the temperature of the convective overshoot region. Therefore, the lithium abundance in AF's could be used a probe for the seat of dynamo if difficulties in the determination of the lithium abundance in AF's can to be overcome. The determination of the lithium abundance is difficult because 1 ) only one group of lithium lines around $6708 \AA$ are observable in the visible range and they are extremely weak, 2) they are mixed up with other lines (Brault and Muller 1975), and 3) they are sensitive to temperature. The techniques of line ratio could be used to eliminate the temperature dependence (Livingston 1988).

\section{STRUCTURE AND DYNAMICS OFARCH FILAMENT SYSTEMS}

The velocity field and dynamics of EFR's in the photosphere has been studied by Born (1974), Zwaan et al. (1985), Brants (1985a, b), and Brants and Steenbeek (1985), and reviewed by Zwaan (1985). In this section, we will emphasize dynamics of AFS's in the chromosphere.

AFS's have been studied by Bruzek $(1967,1969)$, Weart and Zirin (1969), Weart (1970, 1972), Roberts (1970), Frazier (1972), Brants (1985a, b), Zwaan et al. (1985), Chou and Zirin (1988), Alissandrakis et al. (1990), Georgakilas et al. (1990), and Tsiropoula et al. (1992). AFS's trace out the emerging magnetic flux tubes. They show as a set of dark fibrils across the bright plage in $\mathrm{H} \alpha$. Their characteristics are very different from other dark fibrils in the chromosphere, such as field transition arches and filaments. First, AF's accompany emerging flux. Second, their surrounding plage is very bright. Third, they are blueshifted at the tops and redshifted at the ends. The length of AF's is about $20,000-30,000 \mathrm{~km}$, but some can be as long as $40,000 \mathrm{~km}$. The width of individual fibril (AF) is about $1000-3000 \mathrm{~km}$. There is no noticeable change in the width of an AF during its lifetime, once it appears in $\mathrm{H} \alpha$. The lifetime of individual AF in $\mathrm{H} \alpha$ is about $10-30$ minutes, but the entire AFS lasts as long as magnetic flux emerges. The AF's fade away as material inside drains out, and are replaced by the new AF's emerging from below. The AF's continue to form as long as magnetic flux keeps emerging, which sometimes lasts for days. Usually the AF's continue to form in between two spots as they move apart. It is shown in the off-band $\mathrm{H} \alpha$ that the most prominent AF's are connected with growing umbrae (Zirin 1985). Sometimes AFS's emerge in different orientations and slightly different locations to form a complicated active region.

The fact that an AF on the disk is blue-shifted at the tops and red-shifted at the ends suggests that the material inside the tube is moving downward along the tube while the whole tube is ascending. This picture is consistent with the observation of the proper motion of AF's near the limb which shows that AF's are rising up (Bruzek 1967; Chou and Zirin 1988). For an AF on the disk, although its ascending velocity and downward velocity can be estimated by off-band $\mathrm{H} \alpha$ filtergrams (Bruzek 1967), they are better determined by $\mathrm{H} \alpha$ spectra. The $\mathrm{H} \alpha$ spectra show a comma shape if the slit is placed along the AF (Bruzek 1969; Roberts 1970, Chou and Zirin 1988). From the shift of the spectral line, the ascending velocity at the tops of the tube and the downward velocity of the material at footpoints can be determined if the AF is near the 
disk center. However, since an $\mathrm{AF}$ is only opaque in the $\mathrm{H} \alpha$ line center, it is not a trivial to accurately determine its velocities (Chou and Zirin 1988). Some authors (Georgakilas et al. 1990; Tsiropoula et al. 1992) use Beckers' cloud model to derive the velocity structure of AF's.

For the line-of-sight component of the downward velocities at the footpoints in the chromosphere, all observations give rather consistent values, between 35 and $50 \mathrm{~km} \mathrm{~s}^{-1}$. For an AF near or above the limb, its ascending velocity can be determined simply from their proper motions. Yet the results for the ascending velocities of the tops of AF's from the spectroscopic and limb measurements range from $5 \mathrm{~km} \mathrm{~s}^{-1}$ to $20 \mathrm{~km} \mathrm{~s}^{-1}$ in the chromosphere and corona. The discrepancy might be due to that the different AF's are observed at the different heights. At a height of $10,000 \mathrm{~km}$, the velocity is about $10-15$ $\mathrm{km} \mathrm{s}^{-1}$ from the limb observations.

For an AF on the disk, the redshifts of the footpoints last as long as the lifetime of the AF, while the blueshifted material fades before the AF disappears. The fact that the blueshifts do not decrease confirms that the top of the flux tube keeps rising at the end of its life, as is seen indeed in limb observations. The lifetime of 20 minutes for an AF is consistent with the calculated draining time of its material (the draining time of $100-200$ seconds in Section V of Chou and Zirin 1988 was a misprint; it should read $1000-2000$ seconds).

As the magnetic flux tubes rise through the photosphere, they show as the disturbance in the granulation pattern: the aligned dark elongated features parallel to the AFS (Loughhead and Bray 1961; Bray and Loughhead 1964; Brants and Steenbeek 1985; Wang and Zirin 1992). The upward flow in the photosphere near the neutral line of two opposite polarities has been observed. Its velocity can be greater than $0.5 \mathrm{~km} \mathrm{~s}^{-1}$ (Brants 1985b). That the upward motion coincides with the region of transverse magnetic field suggests that the observed upward motion in the photosphere corresponds to the flux tubes breaking through the photosphere. In the photosphere, downward flow of $0.5-2.0 \mathrm{~km} \mathrm{~s}^{-1}$ has been observed in the vicinity of growing pores and sunspots (Gopasyuk 1967, 1969; Kawaguchi and Kitai 1976; Brants 1985b). This downward motion in the photosphere could correspond to the downflow of AF's at their footpoints.

The transverse motion of two footpoints of the AF's also plays an important role in the study of dynamics of the AF's. Chou and Wang (1987) report that the separation velocity of emerging opposite poles in the photosphere is less than $1.2 \mathrm{~km} \mathrm{~s}^{-1}$, based on 24 new bipoles. If AF's indeed connect the opposite poles, the transverse velocity at their footpoints must also be less than $1.2 \mathrm{~km} \mathrm{~s}^{-1}$. Moreover, the limb observations show little or no separation of the two footpoints of an AF seen in vertical projection, while the vertex of the AF ascends (Bruzek 1967; Chou and Zirin 1988). Another evidence for the low separation velocity of footpoints is that the length of an AF on the disk does not increase during its lifetime.

Putting all observational indications together, the scenario of the emergence of flux tubes can be sketched as follows. A flux tube.is brought to the surface by magnetic buoyancy. As it breaks through the photosphere, the rise velocity of vertex is about $0.5 \mathrm{~km} \mathrm{~s}^{-1}$ in the photosphere. The material inside is sliding along the flux tube due to gravity. The line-of-sight component of the downward velocity is about $0.5-2.0 \mathrm{~km} \mathrm{~s}^{-1}$ in the photosphere. The 
flux tube continues to rise into the chromosphere and corona, and show as an $\mathrm{AF}$ in $\mathrm{H} \alpha$. The density of the flux tube is about $10^{10-12} \mathrm{~cm}^{-3}$ (see Section III), which is greater than the ambient density, $\sim 10^{9} \mathrm{~cm}^{-3}$. The downward velocity along the tube has a line-of-sight component of about $35-50 \mathrm{~km} \mathrm{~s}^{-1}$ in the chromosphere, and $0.5-2.0 \mathrm{~km} \mathrm{~s}^{-1}$ in the photosphere. The vertex of the flux tube ascends with a velocity of about $10-15 \mathrm{~km} \mathrm{~s}^{-1}$ in the corona. However, we do not have information on how it is accelerated from $1 \mathrm{~km} \mathrm{~s}^{-1}$ in the photosphere to $10-15 \mathrm{~km} \mathrm{~s}^{-1}$ in the corona. Since the flux tube is visible in $\mathrm{H} \alpha$ only above some level in the chromosphere, we can not use $\mathrm{H} \alpha$ to study its evolution below that chromospheric level.

The material inside would drain out in about 20 minutes, which is the lifetime of an AF. A continuous ascending velocity of $15 \mathrm{~km} \mathrm{~s}^{-1}$ for 20 minutes leads to a height of $18,000 \mathrm{~km}$, which is consistent with the limb observations. The vertex of the flux tube ascends with a velocity of $10-15 \mathrm{~km} \mathrm{~s}^{-1}$, while the two footpoints move apart with a velocity of less than $1.2 \mathrm{~km} \mathrm{~s}^{-1}$ in the photosphere, hence the flux tube is stretched vertically. This poses a challenge for theorists to explain this phenomenon (Chou and Zirin 1988).

Shibata and his colleagues (Shibata 1980; Shibata et al. 1989a, 1989b, 1990) have studied the nonlinear evolution of the undular mode of the magnetic instability for a two-dimensional horizontal flux tube in the solar atmosphere. Their numerical results are consistent with observed quantities, such as rise velocity, downflow velocity, density. However, their solutions are approximately self-similar, and do not show the stretching in the vertical direction. The transverse velocity of the flux tube in the photosphere from their models is about $3-6 \mathrm{~km} \mathrm{~s}^{-1}$, which is much greater than the observed values. In their recent study (Shibata et al. 1992), with the modified lower boundary condition, the transverse velocity of the flux tube can be as small as the observed value.

Recent high-resolution magnetograms show that in the area near neutral lines where the small flux tubes emerge, magnetic polarities are initially highly mixed (Wang and Zirin 1992; Title 1992). The magnetic elements with different polarities move in opposite directions. During the process of segregation, flux cancellation occurs continuously that may trigger sub-flares and surges. These phenomena indicate that the structure and evolution of emergence of magnetic fields is more complicated than the previous simple picture.

\section{PHYSICAL CONDITIONS IN ARCH FILAMENTS}

\subsection{Temperature}

If we had spectra covering several lines formed in an AF, we could derive the temperature and density of the AF. However, since the AF's are optically thick only at $\mathrm{H} \alpha$ line center, it is difficult to deduce their physical conditions from observations against the disk. From good spectra of AF's above the limb, we could deduce their physical conditions, such as the temperature, density, and magnetic field. However, so far our information is very limited.

Chou and Zirin (1988) have discussed the lower and upper limits of AF temperature based on the $\mathrm{H} \alpha$ and D3 images. In $\mathrm{H} \alpha$ the AF's near or above the limb appear brighter than the background, whose brightness 
temperature is about $4200 \mathrm{~K}$. Since the AF's are roughly twice as bright as the background, their excitation temperature must exceed $5000 \mathrm{~K}$, and their kinetic temperature must be still higher because of non-LTE effects. The outer edges of the AF's, which are above the limb, are darker, presumably because of the normal drop in source functions near the edges. The AF's on the disk are dark in $\mathrm{H} \alpha$ against the background because light from the photosphere is scattered.

The upper limit of the AF temperature can be deduced from the fact that the AF's are weak in D3 filtergrams. The He I D3 line is formed by a transition of triplet levels, which requires an excitation temperature of $15,000-20,000$ $K$. Because the AF's show weak absorption in D3, the temperature of AF's can not exceed $15,000 \mathrm{~K}$. However, the intense soft $\mathrm{X}$-ray emission associated with EFR's suggests that the AF's are heated to a temperature higher than $20,000 \mathrm{~K}$ on their way to disappearance. Since the material in an AF starts out below the photosphere, which is about $6000 \mathrm{~K}$, and move fast upward and through the temperature minimum into the chromosphere where it is hotter, the temperature of the AF probably does not drop below $6000 \mathrm{~K}$, and steadily rise toward coronal values.

\subsection{Density}

The material inside an AF is dredged up from below the photosphere and flows down along the flux tube to the footpoints. The downward flow simply means that the material inside the tube does not reach hydrostatic equilibrium, but does not necessarily mean it is denser than its surroundings. The material would drain out in about 20 minutes, the lifetime of the AF. Chou and Zirin (1988) have estimated the density of AF's from the fact that the AF's on the disk are dark in $\mathrm{H} \alpha$ against the background and the AF's near or above the limb appear bright against the background. In the low-density limit, the AF's would be dark against the background because of the pure scattering, and the radiative excitation is more important than the collisional excitation. On the other hand, if the density is high enough, the collisional excitation dominates, and the AF's would be seen as bright against the background. The fact that the AF's are dark against the background on the disk and bright near the limb in $\mathrm{H} \alpha$ indicates that the rate of the collisional excitation from $n=2$ to $n=3$ is comparable to the rate of the radiative excitation by the photons from the photosphere. This leads to the density of the AF's about $10^{11-12} \mathrm{~cm}^{-3}$.

Chou and Zirin (1988) have also used the fact that the AF's are opaque in the $\mathrm{H} \alpha$ center to give a lower limit, about $10^{10}-10^{12} \mathrm{~cm}^{-3}$, for the density of the AF's. In any case, the density of the AF's is higher that the external density, $\sim 10^{9} \mathrm{~cm}^{-3}$.

\subsection{Pressure}

The gas pressure inside the AF is about 0.1 dyne $\mathrm{cm}^{-2}$, if we adopt density $\sim 10^{11} \mathrm{~cm}^{-3}$ and temperature $\sim 8000 \mathrm{~K}$. This value is consistent with the external gas pressure, $\sim 0.3$ dyne $\mathrm{cm}^{-2}$, estimated by using density $\sim 10^{9} \mathrm{~cm}^{-3}$ and temperature $\sim 10^{6} \mathrm{~K}$, at a height of $15,000 \mathrm{~km}$. 


\section{REFERENCES}

Alissandrakis, C. E., Tsiropoula, G., and Mein, P., 1990, Astr. \& Ap., 1990, $230,200$.

Babcock, H. W., 1961, Ap. J., 133, 572.

Born, E. 1974, Solar Phys., 38, 127.

Brants, J. J. 1985a, Solar Phys., 95, 15.

Brants, J. J. 1985b, Solar Phys., 98, 197.

Brants, J. J. and Steenbeek, J. C. M., 1985, Solar Phys., 96, 229.

Brault, J. W. and Muller, E. A., 1975, Solar Phys., 41, 43.

Bray, R. J. and Loughhead, R. E., 1964, "Sunspots" (New York: Dover).

Bruzek, A 1967, Solar Phys., 2, 451.

Bruzek, A 1969, Solar Phys., 8, 29.

Bumba, V. and Howard, R., 1965, Ap. J., 141, 1492.

Chou, D.-Y. and Wang, H., 1987, Solar Phys., 110, 81.

Chou, D.-Y. and Zirin, H., 1988, Ap. J., 333, 420.

Chou, D.-Y. and Fisher, G. H., 1989, Ap. J., 341, 533.

Choudhuri, A. R. and Gilman, P. A., 1987, Ap. J., 316, 788.

Deluca, E. E. and Gilman, P. A., 1987, Ap. J., 316, 788.

Durney, B. R. and Robinson, R. D., 1982, Ap. J., 316, 788.

Fisher, G. H., Chou, D.-Y., and McClymont, A. N., 1989, in "Solar System

Plasma Physics", eds. J. H. Waite, J. L. Burch, and R. L. Moore,

Geophysical Monograph, 54, p. 47.

Frazier, E. N., 1972, Solar Phys., 26, 130.

Galloway, D. J. and Weiss, N. O., 1981, Ap. J., 243, 945.

Georgakilas, A. A., Alissandrakis, C. E., and Zachariadis, TH. G., 1990, Solar Phys., 129, 277.

Gilman, P. A. and Miller, J., 1981, Ap. J., 46, 211.

Gilman, P. A., Morrow, C. A., and Deluca, E. E., 1989, Ap. J., 338, 528.

Glackin, D. L., 1975, Solar Phys., 43, 317.

Golub, L., Krieger, A. S., and Vaiana, G. S., 1976, Solar Phys., 49, 79.

Golub, L., Krieger, A. S., Harvey, J. W., and Vaiana, G. S., 1977, Solar Phys., $53,111$.

Golub, L., Rosner, R., Vaiana, G. S., and Weiss, N. O., 1981, Ap. J., 243, 309.

Gopasyuk, S. I., 1967, Izv. Krymsk. Astrofiz. Obs., 37, 29.

Gopasyuk, S. I., 1967, Izv. Krymsk. Astrofiz. Obs., 40, 111.

Harvey, K. L. and Martin, S. F. 1973, Solar Phys., 32, 389.

Harvey, K. L., Harvey, J. W., and Martin, S. F. 1975, Solar Phys., 40, 87.

Kawaguchi, I. and Kitai, R. 1976, Solar Phys., 46, 125.

Kurokawa, H., 1988, Vistas Astron., 31, 67.

Kurokawa, H., 1991, in "Flare Physics in Solar Activity Maximum 22", eds.

Y. Uchida, R. C. Canfield, T. Watanabe, and E. Hiei, p. 39 (Springer-

Verlag).

Leighton, R. B., 1969, Ap. J., 156, 1.

Livingston, W. C., 1988, private communication.

Loughhead, R. E. and Bray, R. J., 1961, Australian J. of Phys., 14, 337.

Marsh, K. A., 1978, Solar Phys., 59, 105.

Moore, R. L., Tang, F., Bohlin, J. D., and Golub, L., 1977, Ap. J., 218, 286.

Moreno-Insertis, F., 1983, Astr. \& Ap., 122, 241.

Moreno-Insertis, F., 1986, Astr. \& Ap., 166, 291. 
Mosher, J. M., 1977, Ph.D. Thesis, Calif. Inst. of Technology.

Parker, E. N., 1955, Ap. J., 122, 293.

Parker, E. N., 1979, Cosmic Magnetic Fields (New York: Oxford).

Parker, E. N., 1987a, Ap. J., 321, 349.

Parker, E. N., 1987b, Ap. J., 321, 984.

Parker, E. N., 1987c, Ap. J., 321, 1009.

Roberts, P. H. 1970. Thesis, Calif. Inst. of Technology.

Rosner, R., 1980, in "Cool Stars, Stellar Systems, and the Sun", ed. A. K. Dupree, p. 79.

Schmitt, J. H. M. M. and Rosner, R., 1983, Ap. J., 265, 901.

Schoolman, S. A., 1973, Solar Phys., 32, 379.

Schussler, M., 1977, Astr. \& Ap, 56, 439.

Schussler, M., 1979, Astr. \& Ap, 71, 79.

Sheeley, N. R. and Golub, L., 1979, Solar Phys., 63, 119.

Shibata, K 1980, Solar Phys., 66, 61.

Shibata, K, Tajima, T., Matsumoto, R., Horiuchi, T., Hanawa, T., Rosner, R., and Uchida, Y., 1989a, Ap. J., 338, 471.

Shibata, K, Tajima, T., Steinolfson, R. S., and Matsumoto, R., 1989b, Ap. J., 345,584 .

Shibata, K, Nozawa, R., Matsumoto, R., Sterling, A. C., and Tajima, T., 1990, Ap. J., 351, L25-L28.

Shibata, K, Nozawa, R., and Matsumoto, R., 1992, this proceedings.

Spiegel, E. A. and Weiss, N. O., 1980, Nature, 287, 616.

Title, A., 1992, AAS SPD Meeting.

Tsiropoula, G., Georgakilas, A. A., Alissandrakis, C. E., and Mein, P., 1992, Astr. \& Ap., in press.

van Ballegooijen, A. A., 1982, Astr. \& Ap., 106, 43.

Vrabec, D., 1974, in "Chromosphere Fine Structure", ed. R. G. Athay, IAU Symp. 56, p. 201.

Wang, H. and Zirin, H., 1992, Solar Phys., 140, 41.

Weart, S. R., 1970, Ap. J., 162, 987.

Weart, S. R., 1972, Ap. J., 177, 271.

Weart, S. R. and Zirin, H. 1969, Publ. Astron. Soc. Pac., 81, 27 ed. R, G. Athay, p. 161.

Yoshimura, H., 1975, Ap. J., 201, 740.

Zirin, H. 1974, in "Chromosphere Fine Structure", ed. R. G. Athay, IAU Symp. 56, p. 161.

Zirin, H. 1985, Ap. J., 291, 858.

Zirin, H. 1988, Astrophysics of the Sun (Cambridge).

Zwaan, C., 1985, Solar Phys., 100, 397.

Zwaan, C., Brants, J. J., and Cram, L. E. 1985, Solar Phys., 95, 3. 